LNF-96/058 (P)

hep-th/9611018

\title{
Gravitational interaction to one loop in effective quantum gravity円
}

\author{
A. Akhundov ${ }^{a}$ S. Bellucci ${ }^{b}$ A. Shiekh ${ }^{\natural}$ \\ ${ }^{a}$ Universität-Gesamthochschule Siegen, D-57076 Siegen, Germany, and Institute of Physics, \\ Azerbaijan Academy of Sciences, pr. Azizbekova 33, AZ-370143 Baku, Azerbaijan \\ ${ }^{b}$ INFN-Laboratori Nazionali di Frascati, P.O. Box 13, 00044 Frascati, Italy \\ ${ }^{c}$ International Centre for Theoretical Physics, Strada Costiera 11, P.O. Box 586, 34014 Trieste, Italy
}

\begin{abstract}
We carry out the first step of a program conceived, in order to build a realistic model, having the particle spectrum of the standard model and renormalized masses, interaction terms and couplings, etc. which include the class of quantum gravity corrections, obtained by handling gravity as an effective theory. This provides an adequate picture at low energies, i.e. much less than the scale of strong gravity (the Planck mass). Hence our results are valid, irrespectively of any proposal for the full quantum gravity as a fundamental theory. We consider only non-analytic contributions to the one-loop scattering matrix elements, which provide the dominant quantum effect at long distance. These contributions are finite and independent from the finite value of the renormalization counter terms of the effective lagrangian. We calculate the interaction of two heavy scalar particles, i.e. close to rest, due to the effective quantum gravity to the one loop order and compare with similar results in the literature.
\end{abstract}

November 1996

\footnotetext{
${ }^{1}$ PACS number: 04.60.+n

2E-mail addresses: akhundov@azearn.ab.az, bellucci@lnf.infn.it, shiekh@ictp.trieste.it
} 


\section{Introduction}

A longstanding puzzle in quantum physics is how to marry the description of gravity with the field theory of elementary particles. Introducing an effective low-energy theory for processes with a typical energy less the Planck mass, i.e. with $\left|q^{2}\right| \ll M_{\text {Planck }}^{2} \simeq 10^{38} \mathrm{GeV}^{2}$, was suggested by Weinberg [1]. This effective theory could be modeled after the example of Chiral Perturbation Theory [2] for QCD. The important difference to keep in mind, when considering the two cases, is that the analogous of QCD, i.e. a fundamental theory of gravity, is still lacking.

At very low energy, the theory is provided by general relativity. The natural question to address is then the following: what about quantum corrections? There is a problem that arises immediately when trying to answer this question, namely that the $O\left(p^{4}\right)$ constants needed for renormalization are unknown. Indeed, one does not have available experimentally measured quantities, such as the graviton-graviton scattering lengths, in order to determine the finite value of the renormalized counter terms of the effective lagrangian. Donoghue showed first in [3] how to go about this problem and we will handle the matter in a similar way, which explains why we will never treat divergences in the calculation of quantum effects, but only terms with a non-analytic behavior, such as $\ln \left(-q^{2}\right)$ and $1 / \sqrt{-q^{2}}$. We essentially restrict ourselves to calculating only these non-analytic contributions to the scattering matrix elements.

The program consists then in building a realistic model with the particle spectrum of the standard model and renormalized masses, interaction couplings, etc. which include the class of quantum gravity corrections, obtained by handling gravity as an effective theory. This provides an adequate picture at low energies, i.e. much less than the scale of strong gravity (the Planck mass). In this Letter, we start carrying out this program. We begin with scalar fields coupled to quantum gravity and deal with gravity as an effective theory. We calculate the interaction of two heavy scalar particles, i.e. with $m_{1,2} \gg \sqrt{-q^{2}}$, due to the effective quantum gravity to the one loop order. This yields one loop quantum corrections to Newton's potential, which disagree with Donoghue's results of [3, 4. Notice, however, that we do not dispute the validity of the approach, but just the details of the calculation and the results in [3, 4 . Also, we wish to stress that our results hold their validity at low energy, not needing any definite proposal for the complete quantum gravity theory. . $^{-1}$

The outline of this Letter is as follows.

We begin in Section 2 with giving the most general form of the corrections to the Newtonian potential, including the lowest-order correction terms. We also present the Feynman rules in 4 dimensions, discussing the (lack) of importance of the regularization used for the lagrangian. The calculation of the vertex correction is the object of Section 3, where it is described in some detail, in order to make the comparison with Donoghue's calculation and point out the source of the disagreement with it. We also discuss briefly the strategy of our calculation which involves manipulations with the computer. The complete one loop correction to Newton's potential, obtained by adding the contribution of the graviton vacuum polarization, is presented in Section 4. We close the Letter with some concluding remarks, a summary of

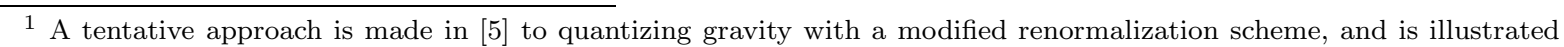
for the example of a massive scalar field with gravity. 
the results obtained and the outlook of possible developments of this investigation.

\section{The lowest-order corrections to the Newtonian potential}

Our notations and conventions on the metric tensor, the gauge-fixed gravitational action, etc. are the same as in [3, 4]. On the grounds of dimensional analysis alone one can anticipate the form of the lowest-order corrections to the Newtonian potential in configuration space [4]:

$$
V(r)=-\frac{G m_{1} m_{2}}{r}\left(1+\alpha \frac{G\left(m_{1}+m_{2}\right)}{r c^{2}}+\beta \frac{G \hbar}{r^{2} c^{3}}+\ldots\right),
$$

which includes the lowest-order relativistic correction, and the lowest-order quantum correction (also relativistic). These power law corrections come from momentum space (where we normally calculate), via the Fourier transforms 3, 4]:

$$
\int \frac{d^{3} q}{(2 \pi)^{3}} e^{-i \vec{q} \cdot \vec{r}} \frac{1}{\sqrt{\vec{q}^{2}}}=\frac{1}{2 \pi^{2} r^{2}}
$$

and

$$
\int \frac{d^{3} q}{(2 \pi)^{3}} e^{-i \vec{q} \cdot \vec{r}} \ln \left(\frac{\vec{q}^{2}}{\mu^{2}}\right)=-\frac{1}{2 \pi^{2} r^{3}} .
$$

Notice that the Fourier transform in (3) seems badly defined, since it is affected by a $\delta^{3}(r)$ ambiguity. Indeed the $\delta$-terms correspond to point-like interactions, which we are not interested in. We are instead interested in the leading quantum effects, i.e. the non-analytic contributions, which dominate at long distance (see the discussion after eq. (4) of [3]). Since these dominant (at large $r$ ) effects are insensitive to the addition of point-like interactions to the lagrangian, the $\delta^{3}(r)$ ambiguity in (3) is completely irrelevant for our calculation.

The importance of these transforms, is that they are from non-analytic terms in momentum space and so cannot be renormalized into the original Lagrangian, and as such one might anticipate that they are of finite magnitude. Because of this, the problem of renormalizing quantum gravity is put off.

Now there is a further magic in the second transform, where the scale $\mu$ does not feed through to configuration space. So this particular calculation is free of the ambiguities normally intrinsic to quantum field theory.

\subsection{The lowest-order Feynman rules}

In gravity there are an infinite number of Feynman rules, although this does not cause a problem, since only a finite number are used when working to any finite order. The lowest-order ones we list as [4:

- The graviton propagator:

$$
\sim \sim \sim \sim D_{\mu \nu \alpha \beta}(p)=\frac{i}{p^{2}+i \varepsilon} P_{\mu \nu \alpha \beta}
$$


- The scalar propagator:

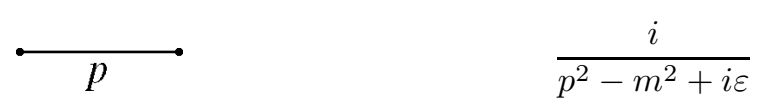

- The scalar-graviton vertex:

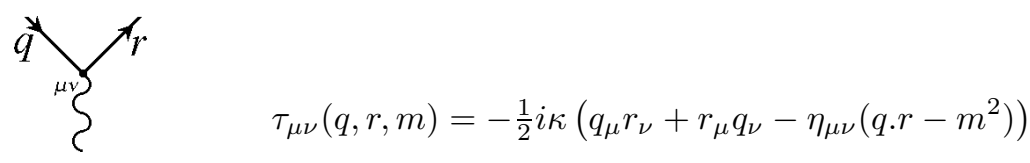

- The scalar-two graviton vertex:

$$
\int_{2 \lambda \tau_{\eta \lambda \rho \sigma}(q, r, m)=\frac{1}{2} i \kappa^{2}\left(\begin{array}{c}
I_{\eta \lambda \alpha \delta} I_{\beta \rho \sigma}^{\delta}\left(q^{\alpha} r^{\beta}+r^{\alpha} q^{\beta}\right) \\
-\quad \frac{1}{2}\left(\eta_{\eta \lambda} I_{\rho \sigma \alpha \beta}+\eta_{\rho \sigma} I_{\eta \lambda \alpha \beta}\right) r^{\alpha} q^{\beta} \\
-\frac{1}{2}\left(I_{\eta \lambda \rho \sigma}-\frac{1}{2} \eta_{\eta \lambda} \eta_{\rho \sigma}\right)\left(q . r-m^{2}\right)
\end{array}\right)}^{\text {where } I_{\mu \nu \alpha \beta} \equiv \frac{1}{2}\left(\eta_{\mu \alpha} \eta_{\nu \beta}+\eta_{\mu \beta} \eta_{\nu \alpha}\right)}
$$

- The three graviton vertex:

$$
\begin{aligned}
& k \underbrace{\sum_{\gamma}^{\alpha \beta} \delta}_{\mu \nu \delta q} \\
& \tau^{\mu \nu}{ }_{\alpha \beta \gamma \delta}(k, q)=
\end{aligned}
$$

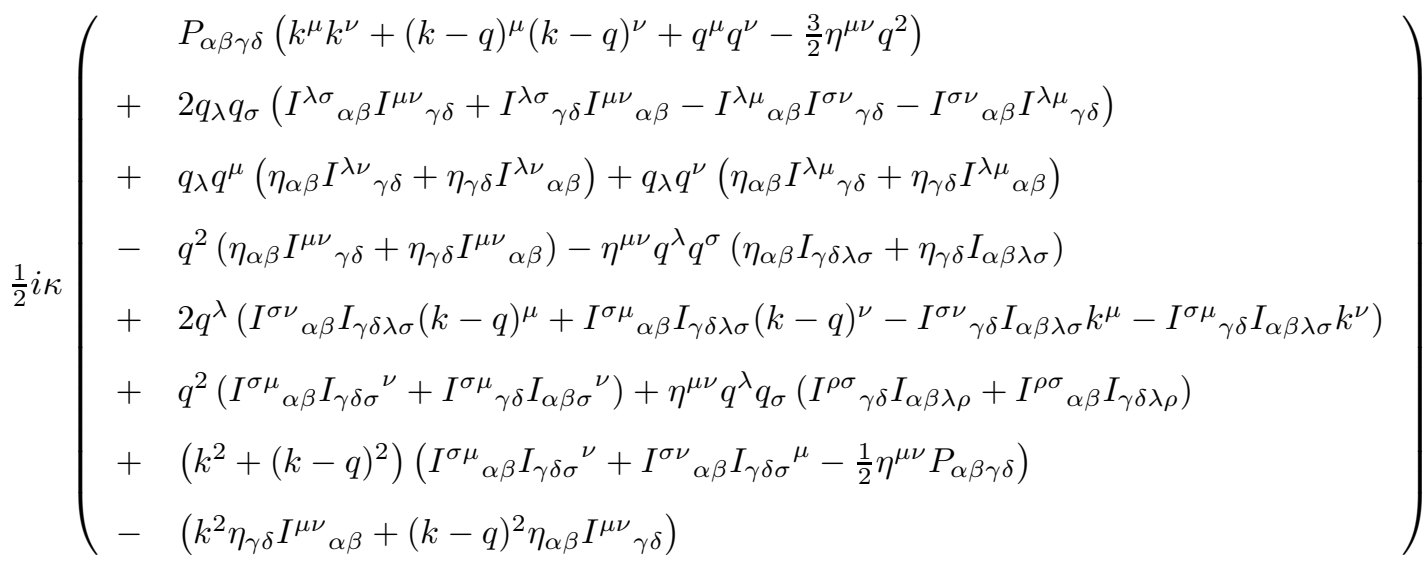

These Feynman rules are presented in 4 dimensions, which would be a problem if we anticipate using dimensional regularization later. However, since we are not dealing with infinite parts at all in this particular calculation, it will not make a difference. Further, there does exist, in the form of operator 
regularization [6], a method not departing from 4 dimensions, and the presented rules are then complete if one were to proceed using this technique. This technique is fundamentally an analytic continuation, and a generalization of the one-loop zeta function technique. One regulates the divergent piece using:

$$
\Omega^{-m}=\lim _{\varepsilon \rightarrow 0} \frac{d^{n}}{d \varepsilon^{n}}\left(\left(1+\alpha_{1} \varepsilon+\ldots+\alpha_{n} \varepsilon^{n}\right) \frac{\varepsilon^{n}}{n !} \Omega^{-\varepsilon-m}\right)
$$

(the alphas being ambiguous)

We have taken the liberty to write down the most general form of this equation, which is normally presented in a more specialised form. We do this, as the difference is critical when it comes to quantum gravity.

\section{$3 \quad$ Vertex corrections}

Only two one-loop vertex contractions contribute non-analytic terms, and they are:

- The graviton loop vertex correction (1a):

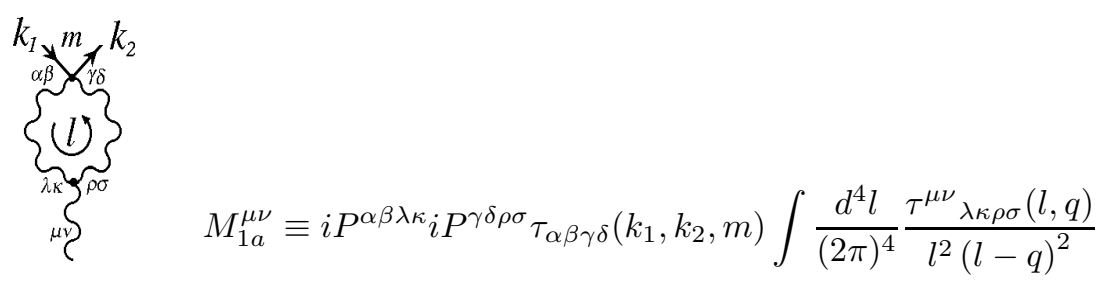

where $q \equiv k_{1}-k_{2}$.

After performing the tensor algebra on a computer, using the Ricci package [7] under the Mathematica [8] program, one arrives at:

$$
M_{1 a}^{\mu \nu}=\frac{\kappa^{3}}{4} \int \frac{d^{4} l}{(2 \pi)^{4}} \frac{\left(\begin{array}{l}
\left(2\left(k_{1}^{\mu} k_{2}^{\nu}+k_{1}^{\nu} k_{2}^{\mu}\right)-g^{\mu \nu} k_{1} \cdot k_{2}\right) l^{2} \\
+\left(5 m^{2}-3 k_{1} \cdot k_{2}\right)\left(2 l^{\mu} l^{\nu}-q^{\mu} l^{\nu}-q^{\nu} l^{\mu}\right) \\
+\frac{g^{\mu \nu}}{2}\left(3 k_{1} \cdot k_{2}-7 m^{2}\right) q^{2}+3\left(2 m^{2}-k_{1} \cdot k_{2}\right) q^{\mu} q^{\nu}
\end{array}\right)}{l^{2}(l-q)^{2}} .
$$

Using the table of integrals [4] for the non-analytic contribution, yields:

$$
M_{1 a}^{\mu \nu}=\frac{\kappa^{3}}{4} \frac{i}{32 \pi^{2}}\left(-\frac{L}{3}\right)\left(26 m^{2}-12 k_{1} \cdot k_{2}\right)\left(q^{\mu} q^{\nu}-g^{\mu \nu} q^{2}\right)
$$

where $L \equiv \ln \left(-q^{2} / \mu^{2}\right)$.

This would agree with Donoghue if we were to drop the $k_{1} \cdot k_{2}$ term. Finally, going on shell using $k_{1} \cdot k_{2}=m^{2}-q^{2} / 2$ yields:

$$
M_{1 a}^{\mu \nu}=-\frac{i \kappa^{3}}{64 \pi^{2}} \frac{7 m^{2}+3 q^{2}}{3}\left(q^{\mu} q^{\nu}-g^{\mu \nu} q^{2}\right) L
$$


Hence onto the form factors:[

$$
V_{1 a}^{\mu \nu}=\frac{\kappa^{2}}{32 \pi^{2}} \frac{7 m^{2}}{3}\left(q^{\mu} q^{\nu}-g^{\mu \nu} q^{2}\right) L
$$

- The graviton-scalar loop vertex correction (1b):

$$
M_{1 b}^{\mu \nu}=i P^{\alpha \beta \lambda \kappa} i P^{\gamma \delta \rho \sigma} i \int \frac{d^{4} l}{(2 \pi)^{4}} \frac{\tau_{\alpha \beta}\left(k_{1}, k_{1}-l, m\right) \tau_{\gamma \delta}\left(k_{1}-l, k_{2}, m\right) \tau^{\mu \nu}{ }_{\lambda \kappa \rho \sigma}(l, q)}{l^{2}(l-q)^{2}\left(\left(l-k_{1}\right)^{2}-m^{2}\right)} .
$$

Doing the algebra on the computer, using the integral tables 迎, going on shell, and working to leading order in $q^{2} / m^{2}$, yields:

$$
\begin{array}{r}
M_{1 b}^{\mu \nu}=\frac{i \kappa^{3}}{64 \pi^{2}}\left(\frac{q^{2}}{16}(20 L+S)\left(k_{1}^{\mu} k_{2}^{\nu}+k_{1}^{\nu} k_{2}^{\mu}+q^{2} \frac{g^{\mu \nu}}{2}\right)+\frac{7 m^{2}}{24}(8 L+3 S)\left(q^{\mu} q^{\nu}-g^{\mu \nu} q^{2}\right)\right) \\
\text { where } S \equiv \pi^{2} m / \sqrt{-q^{2}}
\end{array}
$$

Hence onto the form factors

$$
V_{1 b}^{\mu \nu}=-\frac{\kappa^{2}}{32 \pi^{2}}\left[\frac{q^{2}}{16}(20 L+S)\left(k_{1}^{\mu} k_{2}^{\nu}+k_{1}^{\nu} k_{2}^{\mu}+q^{2} \frac{g^{\mu \nu}}{2}\right)+\frac{7 m^{2}}{24}(8 L+3 S)\left(q^{\mu} q^{\nu}-g^{\mu \nu} q^{2}\right)\right]
$$

Adding (17) with (14) and including also the lowest-order (tree-level) contribution, which can be obtained from (6)

$$
V_{0}^{\mu \nu}=k_{1}^{\mu} k_{2}^{\nu}+k_{1}^{\nu} k_{2}^{\mu}+q^{2} \frac{g^{\mu \nu}}{2}
$$

yields

$$
\begin{aligned}
V^{\mu \nu} & =V_{0}^{\mu \nu}+V_{1 a}^{\mu \nu}+V_{1 b}^{\mu \nu} \\
& =\left(1-\frac{\kappa^{2}}{32 \pi^{2}} \frac{q^{2}}{16}(20 L+S)\right)\left(k_{1}^{\mu} k_{2}^{\nu}+k_{1}^{\nu} k_{2}^{\mu}+q^{2} \frac{g^{\mu \nu}}{2}\right) \\
& -\frac{\kappa^{2}}{32 \pi^{2}} \frac{7 m^{2}}{8} S\left(q^{\mu} q^{\nu}-g^{\mu \nu} q^{2}\right) .
\end{aligned}
$$

This yields the following contribution to the gravitational interaction of two heavy particles (i.e. with $\left.m_{1,2}^{2} \gg\left|q^{2}\right|\right):$

$$
-\frac{\kappa^{2}}{4} \frac{1}{2 m_{1}} V_{\mu \nu}^{(1)}\left[i D^{\mu \nu \alpha \beta}\right] V_{\mu \nu}^{(2)} \frac{1}{2 m_{2}}=-\frac{\kappa^{2}}{4} \frac{m_{1} m_{2}}{2} i\left[\frac{1}{q^{2}}-\frac{\kappa^{2}}{32 \pi^{2}}\left(\frac{5}{2} L+\frac{\pi^{2}\left(m_{1}+m_{2}\right)}{2 \sqrt{-q^{2}}}\right)\right] .
$$

In general, the Feynman rules are large and the tensor algebra immense. Much of the calculational simplicity is restored by submitting this part of the complexity to the computer. However, the intermediate results can be so extensive that even a super-computer can choke without help. For example,

\footnotetext{
${ }^{2}$ Henceforth we neglect further non-analytic terms containing higher powers of $q^{2} / \mathrm{m}^{2}$, since they do not contribute to the leading physical effect we calculate, i.e. the leading quantum corrections to the gravitational interaction of two heavy particles.
} 
imagine one had the contraction of three tensors: $\alpha^{\mu \nu} \beta^{\rho \sigma} \gamma_{\mu \nu \rho \sigma}$ each of which consists of many terms. Then the computer, in trying to contract out the indices, tends to expand out the entire expression, which can easily lead to thousands of terms in the intermediate expression, and so overpower the computers memory. The resolution lies in asking the computer to initially expand out only $\alpha$ for example: $\left(\alpha_{1}^{\mu \nu}+\alpha_{2}^{\mu \nu}+\ldots\right) \beta^{\rho \sigma} \gamma_{\mu \nu \rho \sigma}$. In this way the computer is presented with several terms that can each be contracted separately. This seemingly innocuous move can make all the difference between the machine being able to perform the calculation or not. It is fine details like this that in practice can occupy much of the investigators time.

\section{Putting it all together}

From the counter Lagrangian (5.24) of 't Hooft and Veltman [9], working to second order in the quantum fluctuations $h$ of the gravitational field and using eqs. (3.31), (3.33) of [9], yields the following expression for the contribution of the graviton plus ghost vacuum-polarization Feynman-diagrams

$$
\begin{aligned}
& \Pi_{\alpha \beta \gamma \delta}= \\
& -\frac{\kappa^{2}}{16 \pi^{2}}\left\{\begin{array}{c}
\frac{21}{120} q^{4} I_{\alpha \beta \gamma \delta}+\frac{23}{120} q^{4} \eta_{\alpha \beta} \eta_{\gamma \delta}-\frac{23}{120} q^{2}\left(\eta_{\alpha \beta} q_{\gamma} q_{\delta}+\eta_{\gamma \delta} q_{\alpha} q_{\beta}\right) \\
-\frac{21}{240} q^{2}\left(\eta_{\beta \gamma} q_{\alpha} q_{\delta}+\eta_{\beta \delta} q_{\alpha} q_{\gamma}+\eta_{\alpha \delta} q_{\beta} q_{\gamma}+\eta_{\alpha \gamma} q_{\beta} q_{\delta}\right)+\frac{11}{30} q_{\alpha} q_{\beta} q_{\gamma} q_{\delta}
\end{array}\right\} \text { L+(analytic terms) }
\end{aligned}
$$

where Donoghue's typographical errors have been corrected. This contributes to the two-scalars interaction due to gravitation, as follows:

$$
-\frac{\kappa^{2}}{4} \frac{1}{2 m_{1}} V_{\mu \nu}^{(1)}\left[i D^{\mu \nu \rho \sigma} i \Pi_{\rho \sigma \eta \lambda} i D^{\eta \lambda \alpha \beta}\right] V_{\mu \nu}^{(2)} \frac{1}{2 m_{2}}=-\frac{\kappa^{2}}{4} m_{1} m_{2} i\left(\frac{\kappa^{2}}{32 \pi^{2}}\right)\left(\frac{43}{120}\right) L
$$

Here we neglected terms of order higher than one loop.

Collecting together the one-loop contributions due to the vertex correction (20) and the vacuum polarization (22), we get, after taking the non-relativistic limit $p_{\mu}=(m, 0,0,0), q=(0, \vec{q})$, for the interaction between two scalars

$$
\begin{aligned}
-\frac{\kappa^{2}}{4} \frac{1}{2 m_{1}} V_{\mu \nu}^{(1)}\left[i D^{\mu \nu \alpha \beta}+i D^{\mu \nu \rho \sigma} i \Pi_{\rho \sigma \eta \lambda} i D^{\eta \lambda \alpha \beta}\right] V_{\mu \nu}^{(2)} \frac{1}{2 m_{2}} & =4 \pi G m_{1} m_{2} i\left[\frac{1}{\vec{q}^{2}}\right. \\
& +\frac{\kappa^{2}}{32 \pi^{2}}\left(\frac{107}{60} L+\frac{\pi^{2}\left(m_{1}+m_{2}\right)}{\left.\left.2 \sqrt{\vec{q}^{2}}\right)\right](23)}\right.
\end{aligned}
$$

with $32 \pi G=\kappa^{2}$. This goes over to co-ordinate space as:

$$
V(r)=-\frac{G m_{1} m_{2}}{r}\left[1+\frac{G\left(m_{1}+m_{2}\right)}{r c^{2}}-\frac{107}{30 \pi^{2}} \frac{G \hbar}{r^{2} c^{3}}+\ldots\right],
$$

which agrees with the form anticipated in (1), for the leading corrections to the gravitational interaction of two heavy scalars. The quantum correction agrees with [3, 4] only in size, but not in the details. Notice that the sign of the second term in the r.h.s. of (24) is opposite to Donoghue's result.

\footnotetext{
${ }^{3}$ As observed in [3], the use of dimensionally regularized matrix elements, together with the masslessness of the particles running in the loop, allow us to read off the $L$-term from the divergent contribution due to the vacuum polarization.
} 
As expected, the quantum corrections become large at

$$
r_{0} \simeq \sqrt{\frac{G \hbar}{c^{3}}}=\frac{\hbar c}{M_{P l}}
$$

however in this regime the effective lagrangian approach breaks down. Notice also that the counter terms (which are by definition short-range effects) are expected to contribute only constant terms in (23) (to the one-loop order). The latter reflect themselves in a $\delta^{3}(r)$ contribution to (24), with an unknown coefficient. It is only when the counter terms are inserted as vertices in loop diagrams (i.e. at the two-loop level), that they can contribute to a long-range effect, hence to (23). Finally, at some scale $\mu_{0}$ of the order of (a fraction of) the Planck mass, the constant contributions of the counter terms in (23), which depend on $\mu$ but not on $q$, will have the same size as the $L$-term. Once again, there are, in this energy range, very large corrections to the one-loop result, coming from higher-order terms in the perturbative expansion, which then cannot be trusted any longer.

\section{Conclusion and outlook}

In this Letter we started an investigation aimed at obtaining a class of the quantum corrections to the theory describing elementary particles, e.g. the standard model, due to gravitational effects. Following the pioneering work of Weinberg [1], and stimulated by more recent seminal papers [3, 幽, we choose to treat quantum gravity within the framework of effective field theories. This procedure yields results that are valid at low energy, with respect to the Planck mass. We focus on a theory of scalar fields coupled to the effective quantum gravity and carry out the calculation of the one-loop corrections to the gravitational interaction of two heavy scalars, i.e. close to rest. This requires computing, to the one-loop order, the graviton plus ghost vacuum polarization diagrams, as well as the vertex corrections.

The one-loop corrections are divergent and require the introduction of appropriate renormalizing counter terms in the effective lagrangian to $O\left(p^{4}\right)$. Owing to obvious difficulties in the determination of the finite value of the $O\left(p^{4}\right)$ constants, e.g. relating them to quantities accessible to experiments, the purely analytic terms are not included in the calculation of the quantum corrections [4]. Notice that this sector of quantum corrections is ambiguous, since the purely analytic contributions involve the unknown values of the $O\left(p^{4}\right)$ constants. Hence we calculate that class of $O\left(p^{4}\right)$ corrections yielding effects with a non-analytic behaviour, which are finite and do not depend on the finite value of the renormalized counter terms in the effective lagrangian. This class provides the leading quantum corrections, namely the dominant ones for large $r$ [3].

We have calculated the non-analytic contribution of the vacuum-polarization diagrams to the gravitational scalar-scalar interaction, as in [3, 4], starting from the divergent counter terms of [9]. We performed the calculation of the vertex corrections, as well. They have been calculated before by Donoghue, but we do not agree with his results in 3, 4 . We have checked our calculation, given their complexity and length, with the use of computer programs for algebraic manipulations, based on a package which proved very helpful in dealing with tensors in differential geometry [7], and believe our results are correct. The explicit result for the class of quantum corrections to one loop cannot be measured in experiments, because it is 
too small Nonetheless, it is not arbitrary, rather it follows from the low-energy theory. Whatever the fundamental theory describing the quantum behaviour of gravity, our result must hold its validity at low energies.

Finally, a calculation of the beta functions and anomalous dimensions of a scalar field theory coupled to gravity on a flat background appeared recently [12]. The authors of 12 treat Einstein's theory as an effective field theory. It would be interesting to see if their calculation is relevant, in connection with the effective lagrangian approach to quantum gravity used to obtain our result.

\section{Acknowledgments}

We would like to acknowledge the scientific computer section of ICTP for making hours available on their mainframes. We are grateful to J.F. Donoghue for discussions. A.A. would like to thank the International Centre for Theoretical Physics, INFN-Laboratori Nazionali di Frascati and Technische Hochschule Darmstadt for the hospitality extended to him during the course of this work and for the financial support and the INTAS for the financial support from the project INTAS-93-744. S.B. wishes to thank R. Percacci at SISSA for hospitality when this work was undertaken.

\section{References}

[1] S. Weinberg, in "General Relativity. An Einstein Centenary Survey", ed. by S.W. Hawking and W. Israel, (Cambridge University Press, Cambridge, 1979) p. 790.

[2] S. Weinberg, Physica (Amsterdam) 96A (1979) 327;

J. Gasser and H. Leutwyler, Nucl. Phys. B250 (1985) 465.

[3] J.F. Donoghue, Phys. Rev. Lett. 72 (1994) 2996.

[4] J.F. Donoghue, Phys. Rev. D50 (1994) 3874.

[5] A. Shiekh, Can. J. Phys. 74 (1996) 172.

[6] D. McKeon and T. Sherry, Phys. Rev. Lett. 59 (1987) 532;

ibid. Phys. Rev. D35 (1987) 3854;

D. McKeon, S. Rajpoot and T. Sherry, Phy. Rev. D35 (1987) 3873;

R. Mann et al. Nucl. Phys. B311 (1989) 630;

L. Culumovich et al. Phys. Rev. D41 (1990) 514.

[7] J.M. Lee, "Ricci, A Mathematica package for doing tensor calculations in differential geometry", User's Manual, Version 1.2, 1992-1995.

[8] S. Wolfram, Mathematica (Addison-Wesley, Reading, MA, 1991).

\footnotetext{
${ }^{4}$ The experiments searching for violations of the equivalence principle and deviations from Newton's inverse square law constrain the mass of the Higgs-like boson advocated in extended supergravity theories [10]. These high-precision experiments yield bounds for the antigravity fields appearing in $N=2,8$ supergravity [1].
} 
[9] G. 't Hooft and M. Veltman, Ann. Inst. H. Poincaré A20 (1974) 69.

[10] S. Bellucci and V. Faraoni, Phys. Rev. D49 (1994) 2922.

[11] S. Bellucci and V. Faraoni, Phys. Lett. B377 (1996) 55.

[12] L. Griguolo, R. Percacci, Phys. Rev. D52 (1995) 5787. 